\title{
Generated PSM Web Model for E-learning Platform Respecting n-tiers Architecture
}

\author{
https://doi.org/10.3991/ijet.v12i10.7179 \\ Aziz Srai $\left.{ }^{\bowtie}\right)$, Fatima Guerouate, Naoual Berbiche, Hilal Drissi Lahsini \\ Mohammed V University, Rabat, Morocco \\ aziz.srai.dev@gmail.com
}

\begin{abstract}
E-learning, or learning via a computer or mobile device, is growing. It can take many forms, such as an annotated PowerPoint presentation, a tutorial, or an interactive role-playing game .The possibilities are endless.

Today, $80 \%$ of companies and communities have done a number of interesting and effective e-learning solutions, and 30\% of all professional training are e-learning courses. The development of these platforms is based mainly on different technologies. This technological diversity can make comparing or managing E-learning platforms difficult, and the choice of a given platform will be also complex. Therefore, to address this problem, this paper proposes a solution to generate a PSM model based on n-tier architecture from a PIM model. The language used is the QVT (Query View Transformation) transformation language.
\end{abstract}

Keywords-E-learning, MDA, n-tiers, QVT

\section{Introduction}

In order to cope with the growing complexity and diversity of computer applications to be developed, Research in the field of software engineering has resulted in a Methods and modeling languages. After the paradigm object where everything is object, the community of software engineering has turned towards model-driven engineering (MDE) and the principle of everything is model. It is a case where the application is produced from models. The model-driven engineering has enabled several significant improvements in software development by separating business logic from technical platforms. The main idea of the model-driven engineering is to use the models in the different phases of the development cycle of the application and to provide a set of rules of transformation of models which can lead to the final code in a quasi-automatic way or even automatic.

Model Driven Architecture (MDA) is the model-driven engineering vision of the Object Management Group (OMG). It relies on three types of models. The needs model, called CIM (Computation Independent Model) describes the services to be provided by the application to address the needs of users. The analysis and design model called Platform Independent Model (PIM) defines the structure and behavior of 
the system without indicating the execution platform. The model of the code says, Platform Specific Model (PSM) is the projection of a PIM on a given platform.

This paper is organized as follows: we begin in the first section with an introduction. The section 2 discuss the works that are related to our theme. Section 3 presents the concepts of the MDA architecture (Model Driven Architecture). Section 4 presents our proposed solution to develop E-learning platform respecting the n-tiers architecture. The final section concludes this paper, and outlines future work.

\section{$2 \quad$ Related works}

The goal of the work [1] is to apply the MDA approach on E-learning platforms, authors use UML to model the PIM, as well as ArcStyler for model transformation, then propose a UML profile for EJB. In this paper, the transformation rules between the models were not defined.

The author in [2] proposes a framework based on OMG's Model Driven Architecture. This platform-independent framework specify and classify existing and future Learning Management Systems (LMS).

The work of Bézivin et al. [3] is dedicated to apply the MDA approach for web service platform.

The objective of the work [4] is to generate n-tiers web models using ATL (Atlas transformation language).

The authors in [5] propose a model-driven architecture approach for developing E-Learning Platform with EJB, the transformation rules is not cited.

Recently, the work [6] is arrived to generate a PSM model for codeIgniter framework. The author proposes, as a transformation langauge, the QVT Operational langauge.

\section{MDA architecture}

In order to master software complexity, OMG proposed the MDA approach for the development, integration and maintenance of software-based systems. MDA is a development approach that allows the separation of the functional specifications of a system from the specifications of its implementation on a given technological platform. Thus, MDA is based on several types of models that define different views of a system: CIM, PIM and PSM. It is also based on the transformation of models as a main activity. 


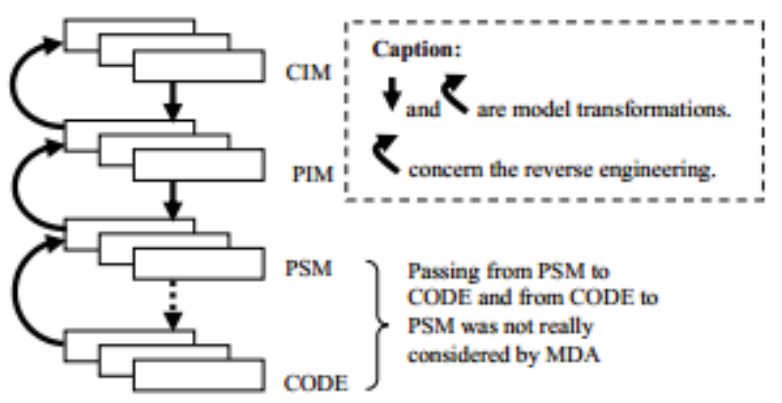

Fig. 1. Global view of MDA

\subsection{Computation Independent Model (CIM)}

The CIM (Computation Independent Model), also known as Business Model or Domain model, describes system requirements and the situation in which the system will be used. It does not represent the details of the structure of the system and remains independent of the technologies of its implementation. This model allows to express the links of traceability with the models which Will be developed in the other phases of the development cycle.

The CIM places the system in its operational context and allows to represent what the system should really ensure as functionalities. In UML, requirements models are represented by use case diagrams. Those models describe, in an abstract way, the different functionalities of the system as well as the actors who interact with this system.

\subsection{Platform Independent Model (PIM)}

The PIM is a high-level abstraction model that is independent of any platform. It describes the system by hiding the details of its use on a particular technological platform. The PIM describes the business logic. He describes software systems as a set of functional entities and their interactions taking into account only the business logic of companies. This model must be clear in order to enable experts in the field to understand it more easily than an implementation model. Thus, they can verify that the PIM is complete and correct more easily.

\subsection{Platform Specific Model (PSM)}

The PSM is a model that is dependent on the technical platform. It is mainly used for the automatic generation of executable code in a given execution environment. The PSM is a representation of the implementation of a particular technology. A PIM can be transformed into one or more PSMs. In fact, for each platform technology, a PSM is generated. 
For the development of PSMs, two approaches are recommended. The first is to use UML profiles to develop PSMs. The use of these profiles has the merit of facilitating PIM to PSM transformations because PIMs and PSMs are both UML models. The second approach consists in the definition of platform-specific metamodels. This approach offers greater freedom in the expression of platforms. However, it has the disadvantage of not facilitating the PIM to PSM transformations.

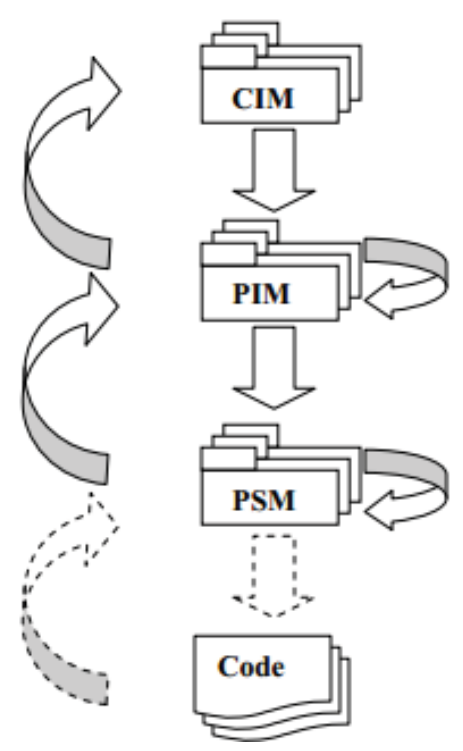

Fig. 2. Levels of abstraction in Model Driven Architecture

Fig. 2. illustrates the main transformations of models defined under the MDA approach.

PIM to PIM transformations aim to enrich, filter or specialize the model during the development cycle without requiring platform dependent information. PIM to PIM transformations are generally used for model refinement.

PSM to PSM transforms apply to a specific model and produce another specific model for the same platform. They are used for the realization and the deployment of components. Code generation, compilation, initialization, and configuration are part of this type of transformation.

PSM-to-PIM transformations must provide an independent model from an existing implementation on a specific platform. These are certainly the most difficult transformations to automate.

PIM to PSM transformations are used when the PIM is sufficiently refined to be projected to an execution platform. 


\section{MDA approach for E-learning platform based on n-tier architecture}

MDA has three modeling levels: Model, Meta-model and Meta-Meta-model. The Meta-Meta-model is the highest abstraction level, it defines the specification language of the Meta-model, and its unique entities. The Meta-model is the level where all instances of a Meta Meta-model are defined, its definition language permits the specification of models. The model level is the lowest one where instances of Metamodels are defined. Therefore, to generate a PSM model for E-learning platform based on n-tier architecture according to this software development method, we have considered the diagram Fig.3. that shows a part of the business use-case model in ELearning.

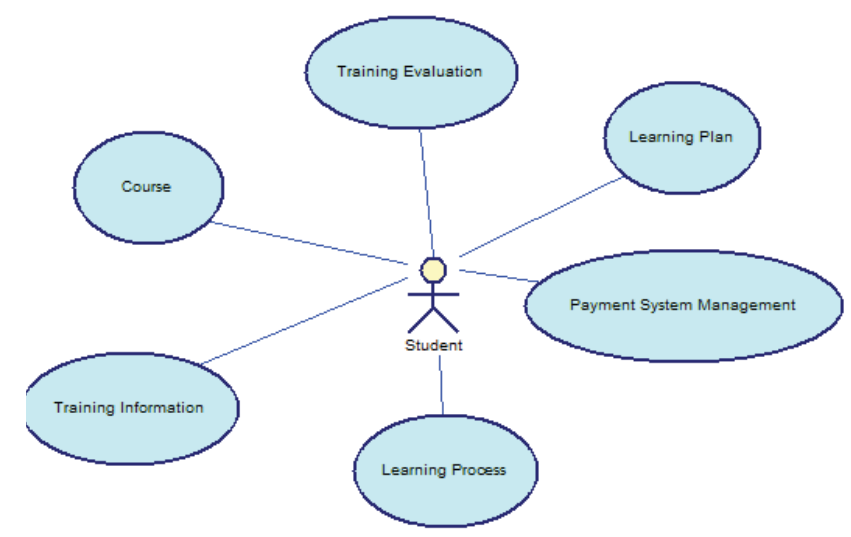

Fig. 3. An extract of use case model of E-Learning System

In practice, extracting a domain model by all open source e-learning platforms such as SCORM, IMS is difficult. However, we can extract a business use case model with LMS (Learning Management System) as an example, which represent the core business model of E-Learning. In this paper, we have considered the student course management system as an example to the development of E-learning platform with the MDA approach, this for letting the paper quite understandable and clear.

We begin by meta-modeling the source UML meta-model presented in the Fig. 4. Next we define the Spring MVC (see Fig.5.), Spring IOC (Spring IOC meta-model are not presented in this paper) and Hibernate DAO (see Fig.6.) frameworks that allow implementation of the CIM, PIM, and PSM meta-models. The CIM model of our work is an UML class diagramme of a case study of a student select course modules (see Fig.7.). The functional model (PIM) is a simplified UML meta-model. The PSM meta-model is composed of the meta-models of Spring MVC, Spring IOC, and Hiberante DAO frameworks. We have defined also the different transformation rules in QVT transformation language, all rules are not cited. However, a part of algorithm that generates action classes and Jsp pages is cited in Fig.8. The result of this work is the n-tiers web model represented in EMF model (see Fig.9). 


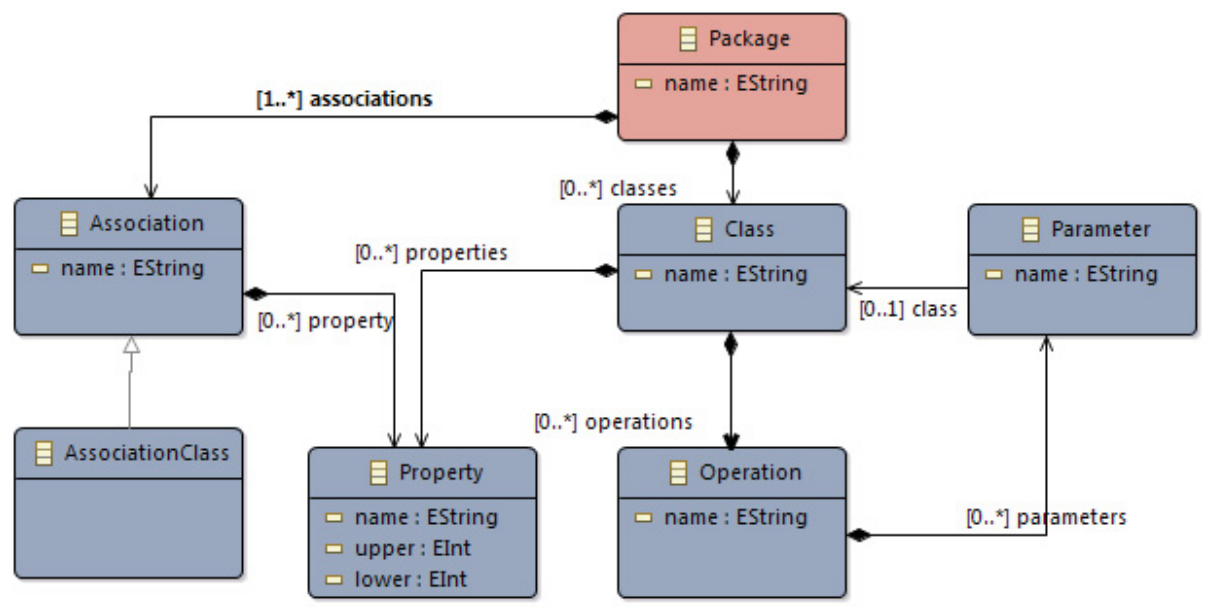

Fig. 4. UML meta-model

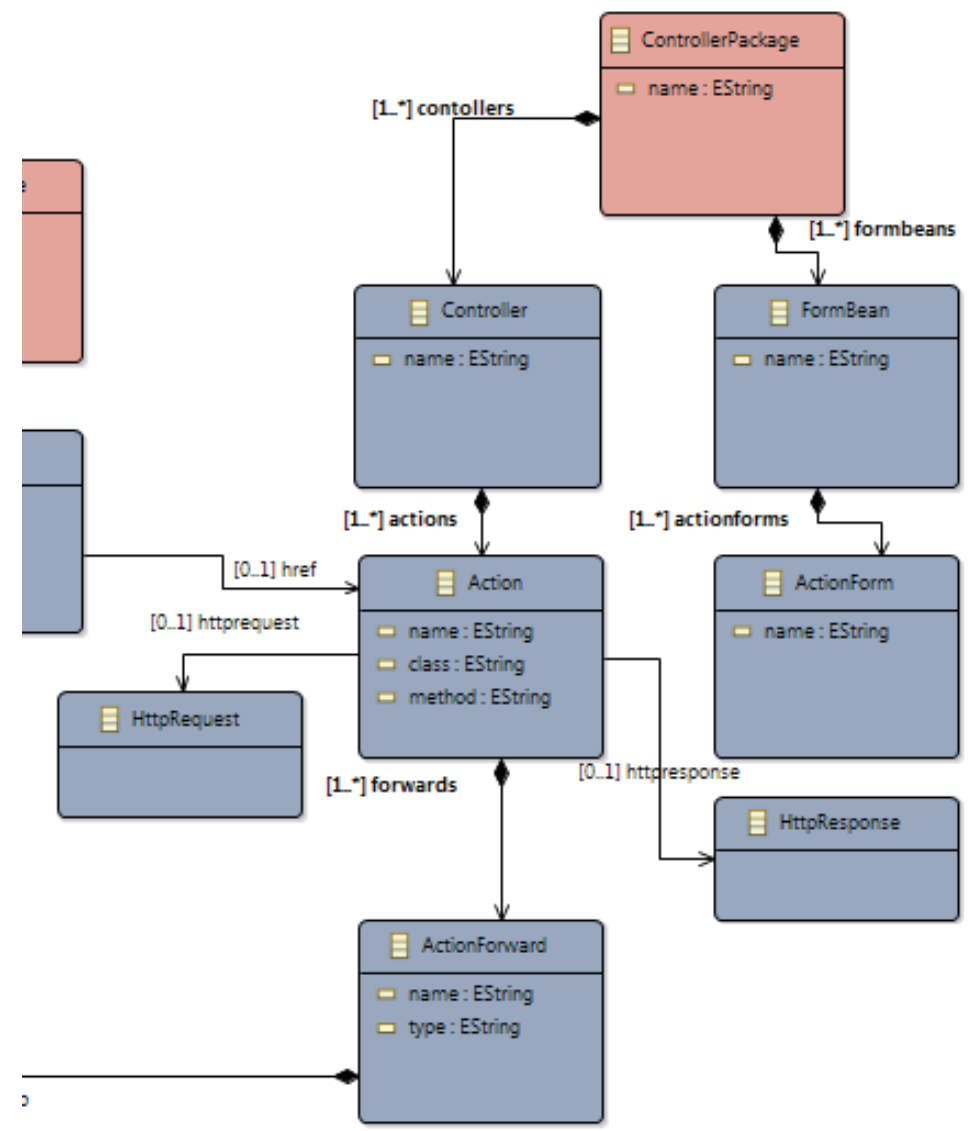

Fig. 5. Part of Spring MVC meta-model 


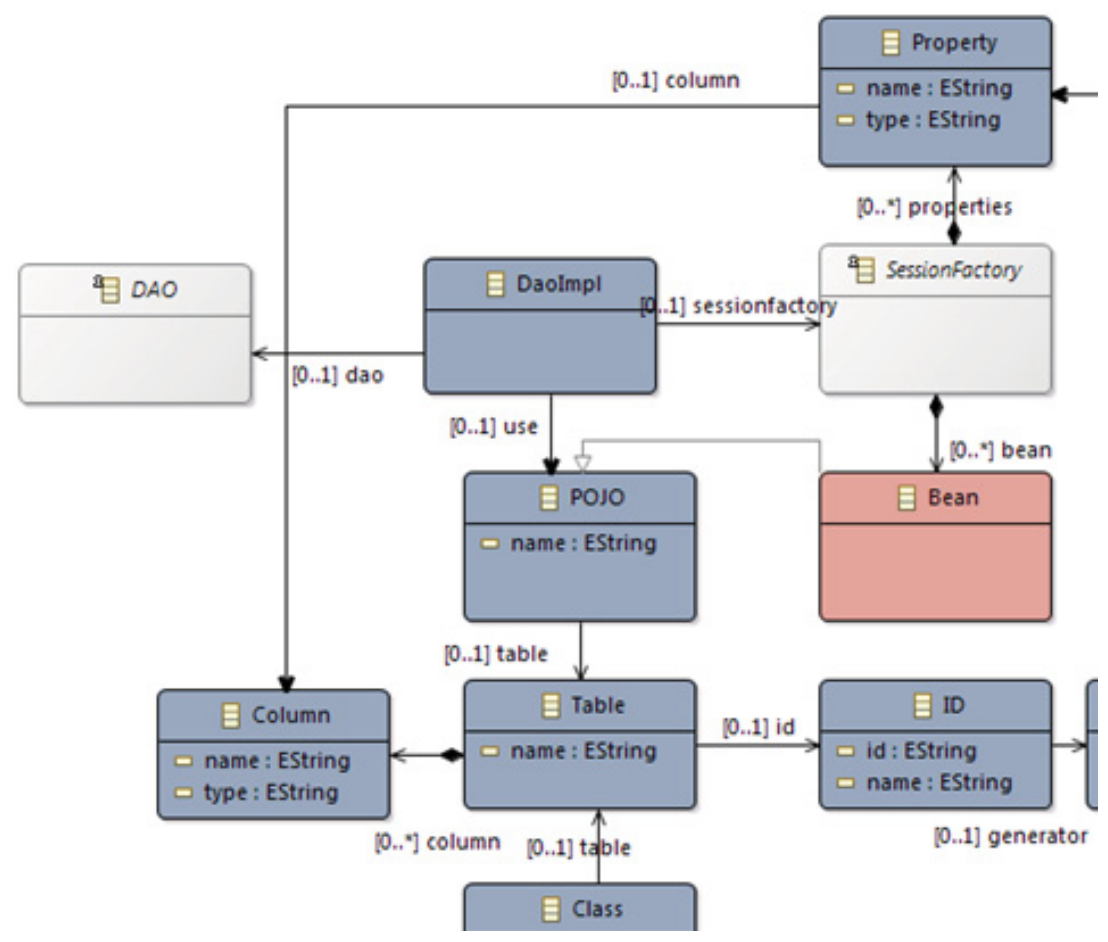

Fig. 6. Part of Hibernate DAO meta-model

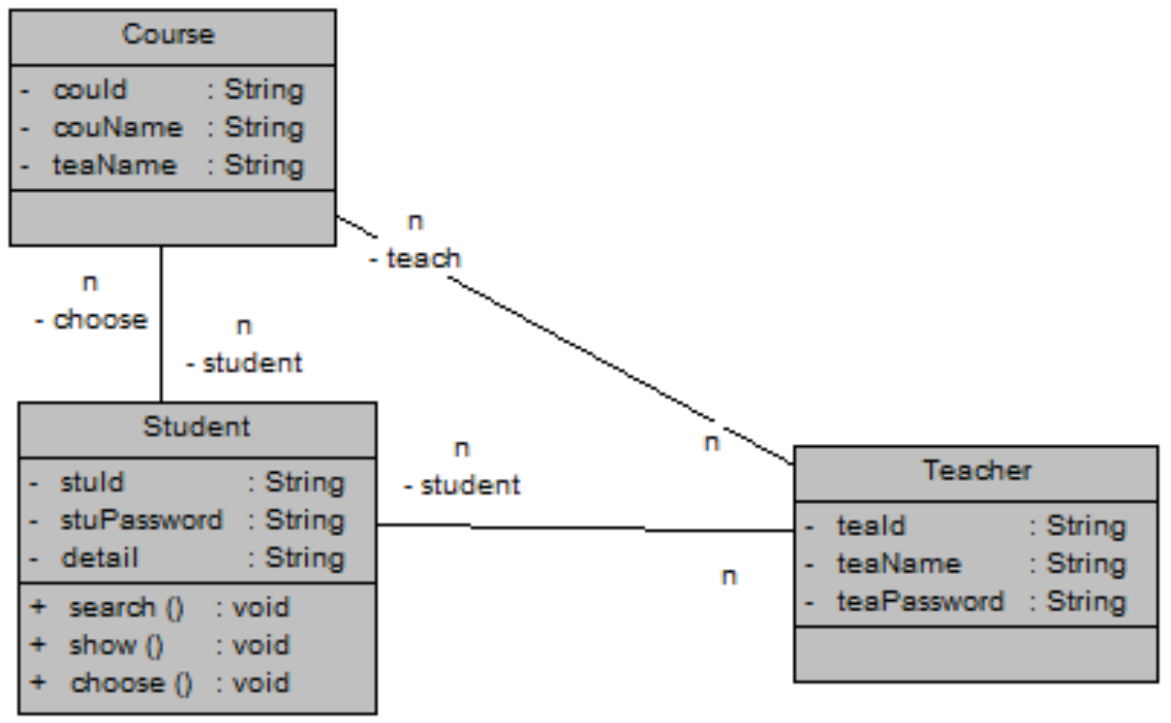

Fig. 7. Class diagram of student select course modules 
[3] Bezivin, J., Hammoudi, S., Lopes, D., Jouault, F., Applying MDA approach for web service platform. In EDOC'04 preceedings of the 8th IEEE International Entreprise Distributed Object Computing Conference, pages 58-70, 2004.

[4] S. Mbarki, M. Rahmouni, MDA Based Modeling and Transformation to Generate N Tiers Web Models. Journal of Software, volume 10, number 3, March 2015. doi:10.17706/jsw.10.3.222-238. https://doi.org/10.17706/jsw.10.3.222-238

[5] X. Zhang et al., A Model-Driven Architecture Approach for Developing E-Learning Platform, (Eds.): Edutainment 2010, LNCS 6249, pp. 111-122, 2010.

[6] A. Srai, et al., MDA approach for CodeIgniter PHP Framework. Proceedings of the $2^{\text {nd }}$ Scientific Day on Computer Science, Optimization and Systems' Modelization (CSOSM'17), pp. 58-61, March 9, 2017, Kenitra, Morocco.

[7] Moreno, N., Romero, J.R.: A MDA-based framework for building interoperable e-learning platforms. Recent Research Development in Learning Technologies (2005).

\section{$7 \quad$ Authors}

Aziz Srai He is a PhD student at Mohamed Mohammed V University in Rabat, Morocco. He received a Master's degree in Computer Science from Hassan II University in Casablanca in 2015. His research interests are MDA, Information Systems and Software Engineering.

Guerouate Fatima is a Research professor at LASTIMI laboratory, Superior School of Technologies of Salé, Mohammed V University in Rabat, Morocco.

Naoual Berbiche is a Research professor at LASTIMI laboratory, Superior School of Technologies of Salé, Mohammed V University in Rabat, Morocco.

Hilal Drissi Lahsini is a Research professor at LASTIMI laboratory, Superior School of Technologies of Salé, Mohammed V University in Rabat, Morocco.

Article submitted 20 May 2017. Published as resubmitted by the authors 25 June 2017. 